\title{
Evolutionary Artificial Neural Networks for Concrete Mix Design Modelling
}

\author{
Stefanus Santosa ${ }^{\# 1}$, Yonathan Purbo Santosa
\#1
Associate Professor, Department of Civil Engineering, Semarang State Polytechnic
University, J1. Prof Sudarto, Semarang, Indonesia, email: stefanus.st@ gmail.com
\#2 Institut für Informatik, Universität Bonn,
Römerstr. 164, Bonn, Germany, email: yonathansantosa@ gmail.com
}

ABSTRACT

Concrete is a composite of materials that each material has complex characteristic, therefore it generate a variety of complicated models. Concrete designer experts are not convinced by the mathematical formula of concrete mix design. According to them, one mix design can only be applied to one mixture. Therefore in every production which involves variations in material characteristics that are slightly different, new mixture design is required. Usually it is done manually with a variety of mixed composition and then test it by using destructive test which has some drawbacks, such as expensive material cost, unpredictable results, and environmentally unfriendly. Moreover concrete mix design nowadays are limited to a few component such as cement, sand, gravel, and water. Therefore it needs to be developed in such way to accommodate recent concrete development. Computational model which is developed in this study uses several parameters such as cement, sand, gravel, water, coal ash (fly ash), and blast furnace slag with Evolutionary Artificial Neural Network approach that based on Genetic Algorithm. Our results proved that the proposed model has lower error rate than Artificial Neural Network model thus help reduce drawbacks that are exist by designing manually.

Key words: Evolutionary Artificial Neural Networks, Genetic Algorithms, Concrete Mix Design.

Corresponding Author: Stefanus Santosa

\section{INTRODUCTION}

Concrete is a composite of materials which every material has complex characteristic, therefore it generates variety of complicated models. Manual and mathematical concrete mixture modelling process which used in vast variety of mixed composition and destructive testing are proved to be inefficient and inaccurate. Therefore, Nikoo applied intelligent computing as a substitute for mathematical model and destructive tests to obtain concrete mix design that is more accurate and less expensive [1].

Concrete mix design is a process of planning the ingredients to achieve correct proportion for desirable compressive strength. In civil engineering field, acquiring concrete mix design is difficult and a sensitive process. It affected by several processing parameters on the quality control of compressive strength. Those parameters play a big role in defining a compressive strength, yet one mixture design is effective only for one compressive strength. In every production which involves variations in material characteristics that are slightly different, new mixture design is necessary. This led to the conventional mixture design results being inaccurate. In the end, it shows that manual mixed design is merely an estimation [2][3]. 
The complexity of the concrete mix design is influenced by variety of components that is used in the design. For high quality and complex behavior concrete requires many parameters to be considered. Material such as cement, sand, gravel, and water as the parameters alone is not enough to produce a good quality concrete. Therefore fly ash, blast furnace slag, clay, rice husk ash, and other additive materials are often added to the mixture.

For instance, lightweight concrete containing coal ash (fly ash and slag concrete - FSC) is a complex material which is difficult to model the behavior [4]. Similarly, according to WenHuan Chine, High-Performance Concrete (HPC) is a highly complex material, which also difficult to model the behavior [5].

From the problem mentioned above, a good model to predict such complex problem are needed. By using smart computing approach (Intelligent Computation), often reliable model can be acquired. This approach is done by trying to mimic human ability to think and solve a problem, which is also called softcomputing. Therefore, this research tries to solve the problems of Civil Engineering by using smart computing hoping that there exist such model that is reliable. This research is focused on the study of intelligent computing constellation for concrete mix design modelling. Studies on this subject have an expeditious development. Following is state of the art for modelling studies of concrete mix design.

Abdurrahmaan Lotfy, et al. create computational models of concrete mixes with clay (Expanded Clay- Lightweight Self-Consolidating Concrete -EC LWSCC). Those concrete mix design is intended to obtain concrete which capable of compacting itself without extra compacting or vibration necessary when casting is done. Modelling was performed using conventional statistical model (regression). This Proposed Mix Design Model, according to researchers, facilitate understanding of the interaction between various parameters on designed concrete [6]. Another model which has developed is a mixed design with elements of blast furnace slag (Furnace Slag- Lightweight Self-Consolidating Concrete -FS LWSCC) using regression approach, general linear model analysis of variance, and backward stepwise technique, in which one of the result was increase in the solution design [7].

Previously, some researchers have tested concrete mix design computational models, both for slump and compressive strength, using linear regression, multiple linear regression, nonlinear regression, Multilayer Perceptron (MLP), Tree Base Modelling [8], SOFM [9], Fuzzy Expert System [10], Adaptive Neural Fuzzy Inference Systems and Fuzzy Inference Systems [11][12], and Artificial Neural Networks (ANN)[13][14][15]. Wen-Huan Chine concluded that ANN-Backpropagation (BPNN) is more accurate in predicting the slump in the value of the concrete mix design comparing to multiple linear regression [5]. The same conclusion of the ANN was also expressed by Ashu Jain, et al [15], that ANN is more accurate in making estimates. Their results, including I-Cheng Yeh [16] showed that ANN is better modelling methods and is able to deliver a more accurate prediction accuracy compared to linear regression, multiple linear regression, and nonlinear regression. I-Cheng Yeh also attempt different approach to model the compressive strength of concrete mix design, which uses Genetic Operation Trees (GOT), ANN, and Non-Linear Regression Analysis (NLRA). The results are, ANN proved to be more accurate than other approaches [17]. Bahador Abolpour developed concrete compressive strength estimation model with Fuzzy Logic approach to show the relationship between the design mix with concrete compressive strength [2]. 
Conventional statistical approaches have been largely abandoned, nevertheless it is still there. Recent developments in concrete mix design modelling studies or State of the Art of this constellation is as delivered by Mehdi Nikoo [1] on Prediction of Concrete Compressive Strength by Evolutionary Artificial Neural Networks. This study involves a Genetic Algorithm (GA) to determine the optimal weights on ANN. GA can improve the performance of some algorithms including Support Vector Machines (SVM), Relevan Vector Machine (RVM), and ANN [18][19][20][21]. The results of GA and ANN algorithm showed that the correlation coefficient value of the parameter compressive strength of concrete at the time of training $=0.910$, testing $=0.935$, and validation $=0.899$. Although this value is quite high but limited to only a few parameters such as cement, sand, gravel, and water. If the parameters added with elements of coal ash (fly ash), blast furnace slag, and other additive materials, the possible values of correlation coefficient can be reduced. Therefore it is necessary to do research which involving complex parameters.

Based on aforementioned description, this research is intended to create computational models of concrete mixed intelligent design with more comprehensive model parameters. This model aims to predict the compressive strength of concrete. This Evolutionary Model was created using the Artificial Neural Network and Genetic Algorithm [22].

The first contribution of this research is crucial to reduce various negative aspects of concrete mix design manual process which are :

1. inefficient because of repeated testing,

2. ineffective because the results of the design is still in the form of estimation,

3. high cost for having to provide materials, equipment, time, and specialized personnel,

4. far from Green Technology principle because destroyed test object is wasted and difficult to decompose.

The second, for industry area, especially readymix concrete industry, such modelling can be implemented to design concrete mixture accurately, robustly, and efficiently in resources usage. Component like cement, sand, gravel, fly ash, blast furnace slag, clay, rice husk ash, water, and other additive materials can be designed using this model to achieve the correct compressive strength without actually create and testing a concrete that is time and resources consuming.

The third is contributing to science development, especially computer science and civil engineering as the new model of intelligent computing model of concrete mix design based on Artificial Neural Networks (ANN) and Genetic Algorithm (GA) with more comprehensive parameter.

\section{MATERIALS AND METHODS}

Based on aforementioned Research Problem, our strategy and research phase to find solution and prove our proposed model is as the following figure. 


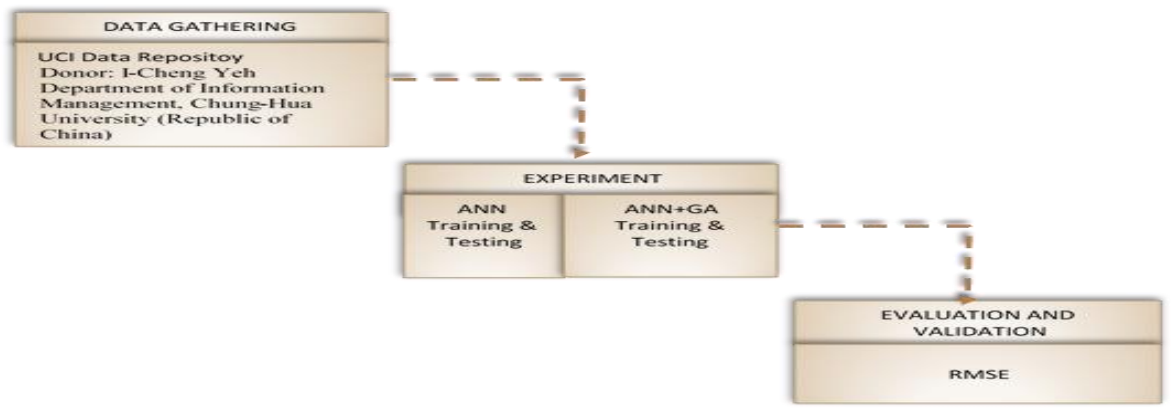

Fig 1: Flow Diagram of Research Method

\section{Data Gathering}

Secondary data were obtained from the UCI Data Repository with characteristics:

1. Data Set Characteristics: Multivariate

2. Attribute Characteristics: Real

3. Number of Instances: 103

4. Number of Attributes: 10

5. Area: Computer

Donor: I-Cheng Yeh, Department of Information Management, Chung-Hua University (Republic of China)

\section{Experiment}

This experiment consist of 3 stages, modelling, training, and testing.

\section{Modelling}

The early models developed and tested on ANN architecture as shown below.

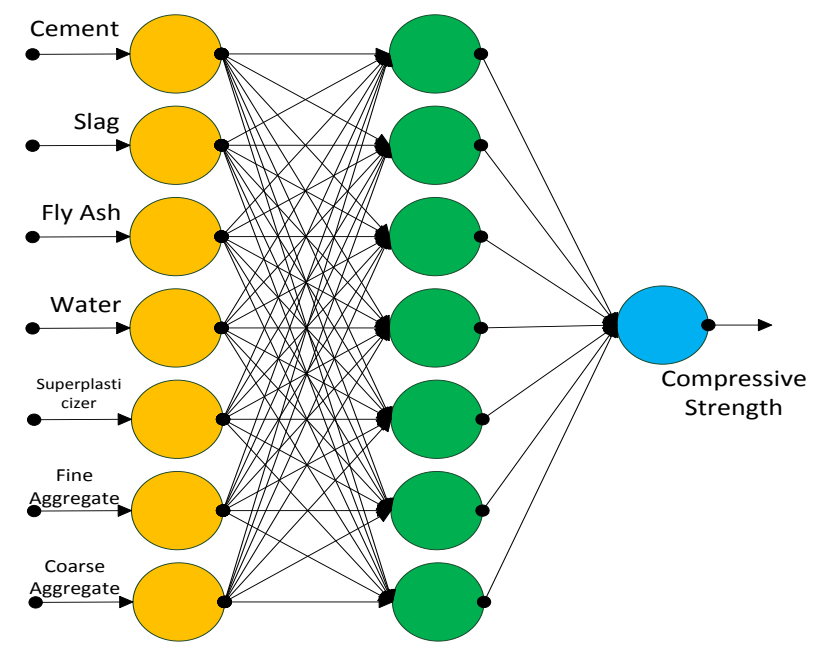

Fig 2: ANN Model Architecture

ANN computational models established by the parameter value Training Iteration, Learning Rate, Momentum, Hidden Neuron, and Number of Hidden Layer. While on ANN with GA optimization based on parameters Number of Generation, Crossover Probability, Mutation Probability, Learning Rate, Population, Hidden Neuron, and Number of Hidden Layer. Experiments were performed repeatedly to obtain a model that has the lowest error.

\section{Training}

The learning process on this network occurs by providing data called training data or training vectors. Training data consists of input and output or input-output which expected in such a 
DOI: https://dx.doi.org/10.26808/rs.ca.i7v5.06

International Journal of Computer Application (2250-1797)

Volume 7- No.5, September-October 2017

way that gained the anticipated output. Parameters involved are Cement, Slag, Fly Ash, Water, Superplasticizer, Coarse Aggregate, Fine Aggregate, and Compressive Strength (28day).

\section{Testing}

After learning gained through training network, the next step is the testing process to test the created models. Inputs such as Cement, Slag, Fly ash, Water, Superplasticizer, Coarse Aggregate, and Fine Aggregate were used to predict the value Compressive Strength (28day)..

\section{Evaluation and Validation Results}

The evaluation of the created models were conducted by measuring the level of error that occurred. The evaluation process was done by using cross validation. Created models can be directly tested by randomly separated data for training and testing.

Error value / RMSE of the two models were compared. Models created with Artificial Neural Networks (ANN) algorithm compared with the models created with the Artificial Neural Networks (ANN) which has been optimized with Genetic Algorithm (GA) by using the same parameters and data.

\section{RESULT AND DISCUSSION}

\section{Experiments with ANN}

This study was divided into two phase. The first phase is design modelling with the Artificial Neural Network (ANN), while the second phase is design modelling with the incorporation of Artificial Neural Network method and Genetic Algorithm (GA) so that the achieved results can be optimized. In this study, the indicators that used to determine prediction error is indicated by the value Root Mean Square Error (RMSE) in each test.

Experiments with Artificial Neural Network begins by setting training iteration $=[100,200$, $\ldots, 1000]$, learning rate $=0.3$, momentum $=0.2$, and the hidden neurons (hidden layer size $)=1$. The number of hidden layers are set constant $=1$. From these experimental results obtained lowest RMSE value is 6.084. RMSE value of 6.084 was obtained in the training iteration parameter value $=100$, learning rate $=0.3$, momentum $=0.2$, and the hidden neurons (hidden layer size $)=1$. Based on RMSE value range which still varied, between 6.084 to 6.648 , then conducting next experiment by changing the learning rate parameter value $=[0.1$, $0.2, \ldots, 1.0]$ while the other parameters constant with the value of the training iteration $=100$ (which chosen because training cycle $=100$ has the best RMSE in previous experiment), momentum $=0.2$, and the hidden neurons (hidden layer size) $=1$ until the lowest RMSE values obtained. Then the next experiment was done by changing momentum parameter values until finally lowest RMSE values was achieved.

Based on the three stages of aforementioned experiments, obtained RMSE values decline significantly starting from 6.084 into 4.943 and the last obtained value is 4.815 . However, subsequent experiments are needed to determine the ideal number of neurons in the hidden layer. Therefore, the next experiment purpose is to determine the value of hidden neurons that vary between $1 \mathrm{sd}$. 10 while training cycle parameter $=100$, learning rate $=0.1$, momentum $=$ 0.1 . The experimental results indicate that the RMSE values stabilized at 4.815 as shown in the following table. 
Table 1. RMSE on Hidden Neuron (hidden layer size) $=1$ to 10 , Momentum $=0.1$, Learning Rate $=0.1$, and the Training Iteration $=100$

\begin{tabular}{|c|c|c|c|c|}
\hline $\begin{array}{c}\text { TRAINING } \\
\text { ITERATION }\end{array}$ & $\begin{array}{c}\text { LEARNING } \\
\text { RATE }\end{array}$ & MOMENTUM & $\begin{array}{c}\text { HIDDEN } \\
\text { NEURON }\end{array}$ & RMSE \\
\hline 100 & 0.1 & 0.1 & $\mathbf{1}$ & 4.815 \\
\hline 100 & 0.1 & 0.1 & $\mathbf{2}$ & 4.902 \\
\hline 100 & 0.1 & 0.1 & $\mathbf{3}$ & 4.979 \\
\hline 100 & 0.1 & 0.1 & $\mathbf{4}$ & 4.819 \\
\hline 100 & 0.1 & 0.1 & $\mathbf{5}$ & 4.984 \\
\hline 100 & 0.1 & 0.1 & $\mathbf{6}$ & 4.935 \\
\hline 100 & 0.1 & 0.1 & $\mathbf{7}$ & 5.14 \\
\hline 100 & 0.1 & 0.1 & $\mathbf{8}$ & 4.855 \\
\hline 100 & 0.1 & 0.1 & $\mathbf{9}$ & 5.137 \\
\hline 100 & 0.1 & 0.1 & $\mathbf{1 0}$ & 5.252 \\
\hline
\end{tabular}

Thus, the smallest RMSE value of predictive models that can be achieved with Artificial Neural Network amounted to 4.185. This model has the best value in the parameter setting training iteration $=100$, learning rate $=0.1$, momentum $=0.1$, hidden layer number $=1$, and the hidden layer size $=1$

\section{Experiments with ANN and GA Optimization}

The second modelling experiments conducted by combining Artificial Neural Networks(ANN) and Genetic Algorithm (GA) methods in order to achieve optimal values. The indicators used to determine the prediction error is also indicated by the value Root Mean Square Error (RMSE) in each test.

Experiments with Artificial Neural Networks and Genetic Algorithm (GA) begins by setting the number of generation $=[50,100,150, \ldots, 500]$, while five other parameters were constant. probability crossover $=0.8$, mutation probability $=0: 10$, learning rate $=5$, population $=100$, and the hidden neurons (hidden layer size) $=8$. The number of hidden layer is set constant $=1$. From this experiment the lowest RMSE result values is 1.389871 as listed in table 2. The RMSE value of 1.389871 is obtained on the value of parameter training cycle $=150$, probability crossover $=0.8$, probability mutation $=0.10$, learning rate $=5$, population $=100$, and hidden neuron (hidden layer size) $=8$.

Based on RMSE value range which is still varied, between 1.389871 to 2.82618 , it is necessary to examine more by changing the value of 5 other parameters which have not be varied, while holding other parameters constant, and so on until all sixth parameter were varied alternately.

Of the six series of experiments that have been conducted, obtained RMSE values was fluctuated, nevertheless the range is imminent. The best RMSE value is 1.389871 as shown in the following table. 
Table 2. RMSE on Number of Generation $=50$ to 150 , Crossover Probability $=0.8$, Mutation Probability $=0.10$, Learning Rate $=5$, Population $=100$, and

Hidden Neuron $=8$

\begin{tabular}{|c|c|c|c|c|c|c|}
\hline $\begin{array}{c}\text { NUMBER } \\
\text { OF GENE } \\
\text { RATION }\end{array}$ & $\begin{array}{c}\text { CROSSOVER } \\
\text { PROB }\end{array}$ & $\begin{array}{c}\text { MUTATION } \\
\text { PROB }\end{array}$ & $\begin{array}{c}\text { LEARN. } \\
\text { RATE }\end{array}$ & $\begin{array}{c}\text { POPU } \\
\text { LATION }\end{array}$ & $\begin{array}{c}\text { HIDDEN } \\
\text { NEURON }\end{array}$ & RMSE \\
\hline $\mathbf{5 0}$ & 0.8 & 0.10 & 5 & 100 & 8 & 2.18202 \\
\hline $\mathbf{1 0 0}$ & 0.8 & 0.10 & 5 & 100 & 8 & 2.092017 \\
\hline $\mathbf{1 5 0}$ & 0.8 & 0.10 & 5 & 100 & 8 & 1.389871 \\
\hline $\mathbf{2 0 0}$ & 0.8 & 0.10 & 5 & 100 & 8 & 1.917591 \\
\hline $\mathbf{2 5 0}$ & 0.8 & 0.10 & 5 & 100 & 8 & 1.623261 \\
\hline $\mathbf{3 0 0}$ & 0.8 & 0.10 & 5 & 100 & 8 & 1.854335 \\
\hline $\mathbf{3 5 0}$ & 0.8 & 0.10 & 5 & 100 & 8 & 1.770221 \\
\hline $\mathbf{4 0 0}$ & 0.8 & 0.10 & 5 & 100 & 8 & 2.538527 \\
\hline $\mathbf{4 5 0}$ & 0.8 & 0.10 & 5 & 100 & 8 & 2.82618 \\
\hline $\mathbf{5 0 0}$ & 0.8 & 0.10 & 5 & 100 & 8 & 1.603296 \\
\hline
\end{tabular}

Based on this value, it can be determined that the best architecture as a predictive model of concrete mix design with Artificial Neural Networks and Genetic Algorithm is with parameter value number of generation $=150$, crossover probability $=0.8$, mutation probability $=0.10$, learning rate $=5$, population $=100$ and hidden neuron $=8$.

When compared with the previous models using Artificial Neural Network in which RMSE value $=4.185$ versus 1.389871 , it is alleged that the researchers prediction are correct, where in neurons weighting in ANN which is done through Genetic Algorithm significantly reduce forecasting errors in concrete mix design.

\section{CONCLUSION}

Modelling of concrete mix design which limited to only a few parameters such as cement, sand, gravel, and water, need to be advanced in order to accommodate recent concrete (technology/product) development. Computational model developed in this study has a parameter of cement, sand, gravel, water, coal ash (fly ash), and blast furnace slag (blast furnace slag) with smart computing approach Artificial Neural Network optimized by Genetic Algorithm (Evolutionary Artificial Neural Network).

Model with Artificial Neural Network approach alone still having high error indicated by RMSE $=4,185$. This study proposes a model with Evolutionary Artificial Neural Network approach with parameter value of number of generation $=150$, crossover probability $=0.8$, mutation probability $=0.10$, learning rate $=5$, population $=100$, and hidden neuron $=8$. This model is intended to minimize the error value of concrete strength prediction on the composition mixture of cement, sand, gravel, water, coal ash (fly ash), and blast furnace slag (blast furnace slag). The experiment results proved that the proposed model has a lower error rate than the prediction model of Artificial Neural Network with RMSE value of 1.389871 compared to 4.185 . 
DOI: https://dx.doi.org/10.26808/rs.ca.i7v5.06

International Journal of Computer Application (2250-1797)

Volume 7- No.5, September-October 2017

\section{ACKNOWLEDGMENTS}

This work has been supported by the Department of Civil Engineering of Semarang State Polythecnic Foundation No: 12759/PL4/PPK/LK/2015 for lecturer research. Authors are also grateful to the UCI Data Repository and Mr. I-Cheng Yeh for providing the datasets.

\section{REFERENCE}

[1] Nikoo, M, Farshid Torabian Moghadam, and Aukasz Sadowski, "Prediction of Concrete Compressive Strength by Evolutionary Artificial Neural Networks", Advances in Materials Science and Engineering Volume 2015, Article ID 849126, 8 pages, Hindawi Publishing Corporation.

[2] Abolpour, B, Benafsheh Abolpour, Roozbeh Abolpour, Hossein Bakhshi, "Estimation of Concrete Compressive Strength by a Fuzzy Logic Model", Science+Business Media B.V, Springer. 2013.

[3] Chandwani V, Vinay Agrawal, Ravindra Nagar, "Applications of Softcomputing in Civil Engineering: A Review", International Journal of Computer Applications (0975-8887), Volume 81 - No 10, November 2013.

[4] Yeh, IC, "Exploring Concrete Slump Model Using Artificial Neural Networks", Journal of Computing in Civil Engineering (C) ASCE / May/June 2006 p.217-221, (2013-Total Cites 1,108 IF=1,385).

[5] Chine, WH, Li Chen, Hsun-Hsin Hsu, Tai-Seng Wang, "Modeling Slump of Concrete Using the Artificial Neural Networks", International Conference on Artificial Intelligence and Computational Intelligence, 2010, p.236-239, IEEE Computer Society.

[6] Lotfy, A, Khandaker M.A. Hossain, Mohamed Lachemi, “Application Of Statistical Models In Proportioning Lightweight Self-Consolidating Concrete With Expanded Clay Aggregates", Construction and Building Materials 65, 2014, 450-469, Elsevier.

[7] Lotfy, A, Khandaker M.A. Hossain, Mohamed Lachemi, "Statistical Models for The Development of Optimized Furnace Slag Lightweight Aggregate Self-Consolidating Concrete", Cement \& Concrete Composite, 55, 2015, 169-185, Elsevier.

[8] Deepa, "Prediction of Compressive Strength of High Performance Concrete Mix Using Tree Base Modelling", International Journal of Computer Applications (0975-8887), Volume 6 - No 5, September 2010.

[9] Nikoo, M., Panam Zarfam, Hassan Sayahpour, "Determination of Compressive Strength Of Concrete Using SOFM", Engineering with Computers, 2015, 31:113-121, Springer-Verlag London.

[10] Neshat M and Ali Adeli, "Design a Fuzzy Expert System to Predict the Concrete Mix Design”, IEEE, 978-1-61284-925-6/11/2011.

[11] Neshat, M.,Ali Adeli,Ghodrat Sepidnam, Mehdi Sargolzaei, 2012, Prediction of Concrete Mix Design Using Adaptive Neural Fuzzy Inference Systems and Fuzzy Inference Systems, Published online: 17 March 2012, Springer-Verlag London Limited 2012.

[12] Tesfamariam S., and H. Najjaran, Adaptive Network-Fuzzy Inferencing to Estimate Concrete Strength Using Mix Design, ASCE_0899-15612007_19:7_550.

[13] Kim JI, Doo Kie Kim, Maria Q. Feng,Frank Yazdani, M, 2004, Application of Neural Networks for Estimation of Concrete Strength, ASCE!0899-1561 2004!16:3 257.

[14] Oztas,Ahmet, Murat Pala, Erdogan Ozbay, Erdogan Kanca,Naci Caglar, M. Asghar Bhatti, 2006, Predicting The Compressive Strength and Slump of High Strength 
Concrete Using Neural Network, Construction and Building Materials 20 (2006) 769775, Elsevier.

[15] Jain, A., Sanjeev Kumar Jha and Sudhir Misra, "Modeling and Analysis of Concrete Slump Using ANN", p 628-633, Journal of Materials in Civil Engineering (C) Asce / September 2008.

[16] Yeh, IC, "Modeling Slump Flow of Concrete Using Second-Order Regressions and Artificial Neural Networks", Cement \& Concrete Composites 29 (2007) p.474-480, Elsevier.

[17] Yeh, IC, Che-Hui Lien, Chien-Hua Peng, Li-Chuan Lien, "Modeling Concrete Strength Using Genetic Operation Trees", Proceedings of the Ninth International Conference on Machine Learning and Cybernetics, Qingdao, 11-14 July 2010, IEEE.

[18] Huang, C and C. Wang, 2006, "A GA-based feature selection and parameters optimization for Support Vector Machines," Expert Systems with Applications, vol. 31, 2006, pp. 231-240. (2013-Total Cites 15,895 IF=1,965)

.[19] Zhao, M, C. Fu, L. Ji, K. Tang, and M. Zhou, 2011, "Expert Systems with Applications Feature selection and parameter optimization for Support Vector Machines : A new approach based on Genetic Algorithm with feature Chromosomes," Expert Systems With Applications, vol. 38, 2011, pp. 5197-5204. (2013-Total Cites 15,895 IF=1,965)

[20] Pendharkar, P.C.,2009, "Genetic Algorithm based Neural Network approaches for predicting churn in cellular wireless network services. Expert Systems with Applications, vol. 36, 2009, pp. 6714-6720. (2013-Total Cites 15,895 IF=1,965).

[21] Awwad, MT, "Developing a Forecasting Model of Concrete Compressive Strength Using Relevance Vector Machines", International Journal of Engineering \& Technology, 3 (2), 2014 224-229, Science Publishing Corporation.s

[22] Chandwani, V., Vinay Agrawal, Ravindra Nagar, "Modeling Slump Of Ready Mix Concrete Using Genetic Algorithms Assisted Training of Artificial Neural Networks", Expert Systems with Applications 42 (2015) 885-893, Elsevier. (2013-Total Cites $15,895 \mathrm{IF}=1,965)$. 